\title{
Gauge Independent Gravitationally-Coupled Electromagnetic Field
}

\author{
C. Aragone* \\ International Atomic Energy Agency and United Nations Educational Scientific \\ and Cultural Organization, International Centre for Theoretical Physics
}

Received October 8, 1971

\begin{abstract}
We give a totally 3-covariant formulation of the gravitationally coupled electromagnetic field (with a source) in terms of physical 3-covariant variables, keeping the correspondence with the A.D.M. formulation of the problem. We introduce a 3covariant time derivation which allows us to discuss the spreading of the transverse and longitudinal components of the electromagnetic field.

Then we present the second-order propagation equation and the Hamiltonian 3covariant equations in a complete electromagnetic gauge independent fashion using the well-known elliptic operator $\Delta\left(g_{i j}\right)$ and we also discuss the dynamical impossibility of disentangling the transverse and longitudinal modes.

Thereafter we extend the 3-covariant formulation to the gravitational field and consider the problems of the initial conditions for the full system, as done by York for the vacuum case.

Finally, we apply the formalism to the merostatic sourceless problem, showing the connection between the merostatic and the static cases.
\end{abstract}

\section{Introduction}

Recently [1] it has been shown in a 4-convariant formalism that one cannot have both a simple propagator and a simple auxiliary condition to determine the gauge.

On the other hand, from a dynamical point of view, it seems very natural to analyse this point, following the lines applied by Arnowitt, Deser and Misner [2] to study the interaction of the electromagnetic field with the tensorial gravitational one; emphasising the role of the physical variables $\left(A_{i}^{T}, \mathscr{E}_{i}^{T}\right)$ of the Maxwell field and the gauge covariance (or invariance) at the different levels of the reduction process.

Thus, in the next sections we shall carry out the process of reduction of the 4-covariant action in a totally 3-covariant way in order to obtain the equation of propagation of the electromagnetic transverse waves in terms of the 3-metric $g_{i j}$. Moreover, we shall study the dynamics and the

* On leave of absence from Instituto de Física, Facultad de Ingeniería, Universidad de la República, Herrera y Reissig 565, Montevideo, Uruguay. 
initial value problem of the full interacting system emphasising conformal techniques. Arguments supporting the introduction of 3-covariant decomposition ( $C$-covariant) against the physically satisfactory noncovariant ( $N$-covariant) have been given by Deser [3], where the underlying 3-metric has been pushed down throughout the formulation of the dynamics of general relativity. In the last two sections we give alternative fully covariant formulations of the "canonical" field equations and we apply them to the merostatic problem of the coupled system, discussing the connection between merostaticity and staticity.

\section{Lagrangian Formulation, $C$-Variables, $C$-Decomposition, and $C$-Time Derivation}

We start from the first-order action for the electromagnetic field given in [2]

$$
I_{M}=\int\left\{A_{\mu, v} \mathscr{F}^{\mu v}+\frac{1}{4} \mathscr{F}^{\mu v} \mathscr{F}^{\alpha \beta} g_{\mu \alpha} g_{v \beta}(-g)^{-\frac{1}{2}}+A_{\mu} \mathscr{J}^{\mu}\right\} d^{4} x
$$

where $A_{\mu}$ is the vector potential, $\mathscr{F}^{\mu v}$ the contravariant skew symmetric field strength density, $\mathscr{J}^{\mu}$ the conserved current density ${ }^{1}$.

We shall consider the $3+1$ decomposition induced on the hypersurfaces $\Sigma \equiv\left\{x^{0}=\right.$ const $\}$ which have the unit normal vector $n_{\mu} \equiv n \delta_{\mu}^{0}$, $n \equiv\left(-g^{00}\right)^{-\frac{1}{2}}$. These hypersurfaces have the induced metric $g_{i j} \equiv_{4} g_{i j}$, and the evolution of the co-ordinates outside $\Sigma$ is determined by the Lagrangian multipliers $N_{i} \equiv g_{0 i}$ [2]. Whenever necessary we shall use the prefix 4 to indicate every four-dimensional quantity, so that all unmarked quantities are understood as being three-dimensional.

Then, for the full metric ${ }_{4} g_{\mu v},{ }_{4} g^{\mu v}\left(N^{i} \equiv g^{i h} N_{h}, g^{i h} g_{h j}=\delta_{j}^{i}\right)$

$$
\begin{aligned}
{ }_{4} g_{00} & =N_{i} N^{i}-n^{2} ; & { }_{4} g^{00} & =-n^{-2}, \\
{ }_{4} g^{0 i} & =n^{-2} N^{i} ; & { }_{4} g^{i j} & =g^{i j}-n^{-2} N^{i} N^{j} .
\end{aligned}
$$

The description of the electromagnetic field will be given in terms of the $\Sigma$-intrinsic quantities ${ }^{2}$

$$
\begin{aligned}
A_{h} & \equiv{ }_{4} A_{h}, & \tilde{A}^{0} & \equiv n_{\mu} A^{\mu}=n \cdot{ }_{4} A^{0}, \\
f_{i j} & \equiv n^{-1} \mathscr{F}_{i j}, & \mathscr{E}^{i} & \equiv g^{i j} \mathscr{F}_{j}^{0}, \\
\dot{\mathcal{J}}_{i} & \equiv n^{-1} \mathscr{J}_{i}, & \varrho & \equiv \mathscr{J}^{0},
\end{aligned}
$$

${ }^{1}\left\{g_{\mu v}\right\}$ has the signature $(-1,+1,+1,+1), R_{\mu v} \equiv \partial_{\alpha} \Gamma_{\mu v}^{\alpha}-\partial_{\mu} \Gamma_{\nu}+\Gamma_{\mu \nu}^{\alpha} \Gamma_{\alpha}-\Gamma_{\mu \beta}^{\alpha} \Gamma_{v \alpha}^{\beta}$.

2 By $\Sigma$-intrinsic quantities we mean geometrical objects under the group of transformations $G_{\Sigma} \equiv\left\{x^{0 \prime}=\varphi^{0}\left(x^{0}\right), x^{i \prime}=\varphi^{i}\left(x^{j}, x^{0}\right)\right\}$ which leave $\Sigma$ invariant

$$
\left(x^{0}=\text { const } \rightarrow x^{0 \prime}=\text { const } t^{\prime}\right) .
$$


which are, respectively, in (3a) a vector and a scalar, in (3b) an antisymmetric tensor and a contravariant vector density; while in (3c) the current is a covariant vector density and the charge $\varrho$ is a density.

Any 3-vector can be decomposed uniquely in a covariant way into its transverse part and its longitudinal part by means of two projection operators $\mathscr{P}^{T}, \mathscr{P}^{L}\left(\nabla_{h}\right.$ is the covariant derivative with respect to the metric $\left.g_{i j}\right)$ :

$$
A_{h}=A_{h}^{T}+A_{h}^{L} \equiv \mathscr{P}_{h l}^{T}(g) A^{l}+\mathscr{P}_{h l}^{L}(g) A^{l},
$$

where

$$
\begin{gathered}
\mathscr{P}_{h l}^{L}(g) A^{l} \equiv \nabla_{h} \Delta^{-1} \nabla_{l} A^{l}, \quad \mathscr{P}_{h l}^{T}(g) A^{l} \equiv\left[g_{h l}-\mathscr{P}_{h l}^{L}(g)\right] A^{l}, \\
\Delta(g) f \equiv g^{-\frac{1}{2}} \partial_{i} \cdot g^{i h} \cdot g^{\frac{1}{2}} \cdot \partial_{h} f .
\end{gathered}
$$

It is worth while mentioning that there exists a "parametrization" of both functional spaces. Every transverse vector can be written as the rotational of some other vector (not in a unique way) and, as is evident in (4b), any longitudinal vector is a gradient of some function. So it is possible to write the decomposition in the form ( $\varepsilon^{i j k}$ is the sign of (ijk) with respect to (123):

$$
A_{h}=g^{-\frac{1}{2}} g_{h i} \varepsilon^{i j k} C_{k, j}+a_{h}=A_{h}^{T}+A_{h}^{L} .
$$

Variation of $a$ over a certain space of functions describes the full space of longitudinal vectors, and free variations of $C_{k}$ give the full space of transverse vectors.

Just as in the action principle there enters a 4-differential $d^{4} x$, so the invariant 4-element of volume $\sqrt{-{ }_{4} g} d^{4} x$ splits into the product of the 3-element of volume $\sqrt{g} d^{3} x$ times the invariant element of line $d \tilde{x}^{0} \equiv n d x^{0}=n_{\mu} d x^{\mu}$, i.e.,

$$
\left(-{ }_{4} g\right)^{\frac{1}{2}} d^{4} x=d \tilde{x}^{0} \wedge g^{\frac{1}{2}} d^{3} x .
$$

In order to achieve a $\Sigma$-covariant formulation of the action principle one needs the $\Sigma$-covariant extension of the non-covariant operator $\partial_{0}$ which allow us to study the whole dynamical system without specifying $\left(n, N_{i}\right)$ as one usually must do (4). First we define the new time derivative (which we denote by $\tilde{\partial}_{0}$ ) for a scalar function $\varphi$ :

$$
\tilde{\partial}_{0} \varphi \equiv n^{-1} \partial_{0} \varphi-N^{i} n^{-1} \partial_{i} \varphi=-n^{\mu} \partial_{\mu} \varphi .
$$

The last expression in (6a) shows that $\tilde{\partial}_{0} \varphi$ is certainly a new scalar under $G_{\Sigma}$. For a covariant vector $V_{i}$, employing the standard [2] momenta for the gravitational field $\left\{\pi_{i j} \equiv \sqrt{g}\left[K_{i j}-g_{i j} K\right] ; K_{i j} \equiv n \cdot{ }_{4} \Gamma_{i j}^{0}\right\}$, 
we extend the above definition thus:

$$
\begin{aligned}
\tilde{\partial}_{0} V_{i} & \equiv-n^{\mu} \nabla_{\mu} V_{i}-K_{i}^{j} V_{j}-\partial_{i} n \cdot V^{0} \\
& =n^{-1} \partial_{0} V_{i}-n^{-1} N^{j} \partial_{j} V_{i}-n^{-1}\left(\partial_{i} N^{j}\right) \cdot V_{j} .
\end{aligned}
$$

The definition shows explicitly that $\tilde{\partial}_{0} V_{h}$ is a covariant vector because it is the sum of three covariant vectors ${ }^{3}$. The second expression of $\tilde{\partial}_{0} V_{h}$ depends only upon the vector $V_{h}$ itself (through its spatial and time derivatives) and on the 4 Lagrange multipliers $\left(n, N^{i}\right)$; there is no reference to the metric $g_{i j}$. At this stage, by application of the Leibniz rule to $\varphi=W^{i} V_{i}$, we obtain $\tilde{\partial}_{0} W^{i}$ (which again does not depend on the induced metric $g_{i j}$ )

$$
\tilde{\partial}_{0} W^{i} \equiv n^{-1} \partial_{0} W^{i}-n^{-1} N^{j} \partial_{j} W^{i}+n^{-1}\left(\partial_{j} N^{i}\right) W^{j} .
$$

(Actually we define $\tilde{\partial}_{0} W^{i}$ by means of $(6 c)$ and then we verify the validity of the Leibniz rule because either $\tilde{\partial}_{0} W^{i}, \tilde{\partial}_{0} V_{i}, \tilde{\partial}_{0} \varphi$ have been defined independently).

Following this procedure one can obtain the value of $\tilde{\partial}_{0} T_{j 1 \ldots j m}^{i 1 \ldots i m}$ for any $m$ and $n$. In the case of a symmetric tensor of rank two:

$$
\tilde{\partial}_{0} t_{i j}=n^{-1} \partial_{0} t_{i j}-n^{-1} N^{l} \partial_{l} t_{i j}-n^{-1} \partial_{j} N^{l} t_{i l}-n^{-1} \partial_{i} N^{l} t_{l j} .
$$

When $t_{i j}=g_{i j}$ Eq. (6d) gives for $\tilde{\partial}_{0} g_{i j}, \tilde{\partial}_{0} g^{i j}$ and $\partial_{0} \sqrt{g}$ :

$$
\begin{aligned}
& \tilde{\partial}_{0} g_{i j}=n^{-1} \partial_{0} g_{i j}-n^{-1}\left(\nabla_{i} N_{j}+\nabla_{j} N_{i}\right)=2 K_{i j}, \\
& \tilde{\partial}_{0} g^{i j}=-2 K^{i j}, \quad \tilde{\partial}_{0} \sqrt{g}=K \sqrt{g}, \\
& \tilde{\partial}_{0}\left[g_{i j} g^{-\frac{1}{2}}\right]=\left[2 \pi_{i j}-\frac{1}{2} \pi g_{i j}\right] \cdot g^{-1} \equiv 2 \hat{\pi}_{i j} g^{-1} .
\end{aligned}
$$

The last Eq. (7b) permits us to extend $\tilde{\partial}_{0}$ to a 3-density $\sigma$. In fact, from the Leibniz rule, we get for the value of $\tilde{\partial}_{0} \sigma$ :

$$
\tilde{\partial}_{0} \sigma \equiv \sigma K+g^{\frac{1}{2}} \tilde{\partial}_{0}\left[\sigma \cdot g^{-\frac{1}{2}}\right]=n^{-1} \partial_{0} \sigma-n^{-1} \partial_{i}\left(N^{i} \sigma\right) .
$$

As $\tilde{\partial}_{0} \sigma d \tilde{x}^{0} \wedge d^{3} x=\left[\partial_{0} \sigma-\partial_{i}\left(N^{i} \sigma\right)\right] d^{4} x$ is an exact co-ordinate divergence times $d^{4} x$, we are able to discard terms of this type in the action in order to obtain the field equations.

Because of Eqs. $(7 \mathrm{a}-\mathrm{b})$ which say that $\tilde{\partial}_{0} g_{i j} \neq 0$, the "time" derivative of a covariant vector $V_{i}$ does not coincide with the "time" derivative of

${ }^{3}$ The first two terms are obviously covariant vector. The last term,

$$
\left(\partial_{h} n\right) V^{0}=\left[\partial_{h} \log n\right]\left(n V^{0}\right)=\left[\partial_{h} \log n\right] \tilde{V}^{0}=\left[\partial_{h} \log n\right]\left(n_{\mu} V^{\mu}\right),
$$

can be regarded as a scalar $\left(n_{\mu} V^{\mu}\right)$ times the covariant vector $\partial_{h} \log n$ since $\log n$ is an intrinsic scalar. 
the contravariant component $V^{i}$ of the same vector, i.e.,

$$
\tilde{\partial}_{0} V^{i}-g^{i j} \tilde{\partial}_{0} V_{j}=\tilde{\partial}_{0} g^{i j} \cdot V_{j} .
$$

Applying the definitions given throughout this section we can write the first-order action in the explicit $C$-form, showing the $p \dot{q}-H$ structure:

$$
\begin{aligned}
I\left(A, \mathscr{E}, f, \tilde{A}^{0}, g_{i j}, n, N^{i}\right)=\int\{ & -\mathscr{E}^{i} \tilde{\partial}_{0} A_{i}-f^{i j} \partial_{i} A_{j}-\frac{1}{2}\left[\mathscr{E}^{i} \mathscr{E}_{i}-\frac{1}{2} f^{i j} f_{i j}\right] g^{-\frac{1}{2}} \\
& \left.+\tilde{A}^{0}\left[\partial_{i} \mathscr{E}^{i}-\varrho\right]+A_{i j} j^{i}\right\} d \tilde{x}^{0} \wedge d^{3} \boldsymbol{x} .
\end{aligned}
$$

In this form we already recognize $\tilde{A}^{0}$ as the Lagrangian multiplier associated with the Maxwell differential constraint $\partial_{h} \mathscr{E}^{h h}=\varrho$. The standard method to "solve" this differential constraint is the $T-L$ decomposition.

As the metric $g_{i j}$ with which we are dealing will not, in general, be flat, and as the $\hat{\partial}_{0}$ operator does not commute with the covariant derivatives $\nabla_{h}$, there will be some modifications in the usual properties which involve $\left[\partial_{h} \log n\right]$ and $\tilde{\partial}_{0} g_{i j}$. Moreover, in the following section, we shall estimate the non-vanishing commutators $\left[\mathscr{P}^{T}, \tilde{\partial}_{0}\right]=-\left[\mathscr{P}^{L}, \partial_{0}\right]$ which tell us that under $\tilde{\partial}_{0}$ - time evolution a transverse (longitudinal) vector generally acquires longitudinal (transverse) parts.

\section{Kinematics of the $T+L$ Decomposition}

In Ref. 3 the orthogonality between a longitudinal vector and a transverse density has been pointed out, i.e.,

$$
\int V_{i}^{L} \mathscr{W}^{T i} d^{3} \boldsymbol{x}=0 .
$$

Now we shall calculate the value of the same products, but integrating over the full 4-space. In this case we have for $V_{i}=V_{i}^{T}+\partial_{i} v$; $\mathscr{W}^{h}=\mathscr{W}^{T h}+\mathscr{W}^{L h}:$

$$
\int V_{i}^{L} \mathscr{W}^{T i} d \tilde{x}^{0} \wedge d^{3} x=-\int v\left(\partial_{i} \log n\right) \mathscr{W}^{T i} d \tilde{x}^{0} \wedge d^{3} \boldsymbol{x}
$$

or equivalently:

$$
\int n^{-1} \partial_{i}[n v] \mathscr{W}^{T i} d \tilde{x}^{0} \wedge d^{3} x=0
$$

because for a contravariant density $\mathscr{W}^{i}$

$$
\int V_{i}^{L} \mathscr{W}^{i} d \tilde{x}^{0} \wedge d^{3} \boldsymbol{x}=-\int v n^{-1} \partial_{i}\left[n \mathscr{W}^{i}\right] d \tilde{x}^{0} \wedge d^{3} \boldsymbol{x}
$$

holds.

Better results are obtained when considering the product $\int \mathscr{W}^{i} \tilde{\partial}_{0} V_{i} d \tilde{x}^{0} \wedge d^{3} \boldsymbol{x}$; in this case orthogonality holds for some mixed inner products. The full set of inner products, with a 4-dimensional 
integration, is:

$$
\begin{gathered}
\int \mathscr{W}^{T}{ }^{2} \tilde{\partial}_{0} V_{i}^{L} d \tilde{x}^{0} \wedge d^{3} \boldsymbol{x}=0, \\
\int \mathscr{W}^{L}{ }_{i} \tilde{\partial}_{0}\left[g^{\frac{1}{2}} V^{T i}\right] g^{-\frac{1}{2}} d \tilde{x}^{0} \wedge d^{3} \boldsymbol{x}=0, \\
\int \mathscr{W}^{L i} \tilde{\partial}_{0} V_{i}^{T} d \tilde{x}^{0} \wedge d^{3} \boldsymbol{x}=2 \int \mathscr{W}^{L i} \hat{\pi}_{i j} V^{T j} g^{-\frac{1}{2}} d \tilde{x}^{0} \wedge d^{3} \boldsymbol{x}, \\
\int \mathscr{W}^{i} \tilde{\partial}_{0} V_{i}^{L} d \tilde{x}^{0} \wedge d^{3} \boldsymbol{x}=-\int \partial_{i} \mathscr{W}^{i} \cdot \tilde{\partial}_{0} v d \tilde{x}^{0} \wedge d^{3} \boldsymbol{x} .
\end{gathered}
$$

The relations (10) can be applied to simplify the last term in the action (8), taking into account the current conservation which holds as a consequence of the 4-covariant field equations $\partial_{\mu} \mathscr{J}^{\mu}=0$.

The $3+1$ version of the current conservation is:

$$
n^{-1} \partial_{h}\left[n j^{h}\right]+\tilde{\partial}_{0} \varrho=0,
$$

and then, by means of $(7 \mathrm{~d}),(10 \mathrm{c})$ and (12), the last term in (8) becomes:

$$
\int\left(A_{i}^{T} j^{i}+A_{i}^{L} j^{i}\right) d \tilde{x}^{0} \wedge d^{3} x=\int\left(A_{i}^{T} j^{i}-\varrho \tilde{\partial}_{0} a\right) d \tilde{x}^{0} \wedge d^{3} x .
$$

Now we come to the problem of transverse and longitudinal mixing modes of propagation which, as we shall see, stems from the lack of commutation between the spatial derivation operator $\hat{\partial}_{i}$ and the time evolution operator $\tilde{\partial}_{0}$.

There are two relations which provide the clue to the mixing problem of a vector. The first concerns the time evolution of a longitudinal vector

$$
\left[\tilde{\partial}_{0}, \partial_{h}\right] f=\left(\partial_{h} \log n\right) \cdot \tilde{\partial}_{0} f,
$$

which can also be written

$$
\tilde{\partial}_{0} \cdot \partial_{h} f=n^{-1} \partial_{h}\left(n \tilde{\partial}_{0} f\right),
$$

showing that the time evolution of a longitudinal vector $\partial_{h} f$ differs by $\left(\partial_{h} \log n\right) \tilde{\partial}_{0} f$ from being longitudinal $l^{4}$. The second gives the connection between the evolution of the divergence of a contravariant vector density and the divergence of the time $\left(\tilde{\partial}_{0}\right)$ derivative of the same object,

or,

$$
\left[\tilde{\partial}_{0}, \partial_{i}\right] \mathscr{W}^{i}=\left(\partial_{i} \log n\right) \tilde{\partial}_{0} \mathscr{W}^{i}
$$

$$
\tilde{\partial}_{0} \partial_{i} \mathscr{W}^{i}=n^{-1} \cdot \partial_{i} \cdot n \cdot \tilde{\partial}_{0} \cdot \mathscr{W}^{i}
$$

which is the same.

In particular, Eqs. (15) show that the time evolution of a transverse vector density $\mathscr{W}^{T i}$ has a non-vanishing source for its longitudinal component $\partial_{i}\left[\tilde{\partial}_{0} \mathscr{W}^{T i}\right]=-\left(\partial_{i} \log n\right) \cdot \tilde{\partial}_{0} \mathscr{W}^{T i}$.

${ }^{4}$ The source of the $T$-component of the time evolution of a longitudinal vector $V_{i}^{L}$ is $S^{\imath}=\varepsilon^{\imath \jmath h} \partial_{j} \hat{\partial}_{0} V_{k}^{L}=\varepsilon^{\ell, k} \cdot \partial_{0} V_{J}^{L} \cdot\left[\partial_{k} \log n\right]$. 
More precisely, we can exhibit the spreading of the transverse and longitudinal components of a vector $V^{i}=(g)^{-\frac{1}{2}} \mathscr{V}^{T i}+g^{i j} \partial_{j} v$ calculation the "mixed" projections, i.e.,

$$
\begin{aligned}
\mathscr{P}_{i j}^{L} \tilde{\partial}_{0} \mathscr{V}^{T j} & =-g^{\frac{1}{2}} \partial_{i} \cdot \Delta_{g}^{-1} \cdot g^{-\frac{1}{2}}\left(\partial_{j} \log n\right) \cdot \tilde{\partial}_{0} \mathscr{V}^{T j}, \\
\mathscr{P}^{T}{ }_{i}{ }_{i} \tilde{\partial}_{0} V_{j}^{L} & =\mathscr{P}^{T}{ }_{i}^{j}\left(\partial_{j} \log n\right) \tilde{\partial}_{0} v \\
& =\left(\partial_{i} \log n\right) \tilde{\partial}_{0} v-\partial_{i} \Delta_{g}^{-1} g^{-\frac{1}{2}} \partial_{j}\left[g^{\frac{1}{2}} g^{j h}\left(\partial_{h} \log n\right) \cdot \tilde{\partial}_{0} v\right],
\end{aligned}
$$

while the $L$-projection of $\tilde{\partial}_{0} V_{i}^{L}$ is:

$$
\mathscr{P}^{L}{ }_{i}^{j} \tilde{\hat{\partial}}_{0} V_{j}^{L}=\partial_{i} \tilde{\partial}_{0} v+\partial_{i} \Delta_{g}^{-1} g^{-\frac{1}{2}} \partial_{j} g^{\frac{1}{2}} g^{j l} \cdot \partial_{l} \log n \cdot \tilde{\partial}_{0} v .
$$

It is interesting to point out the different role played by $n$ with respect to the remaining three Lagrangian multipliers $N^{i}$. It is sufficient to choose co-ordinates so that $n=1$ (not necessarily gaussian) to regain formally all the flat properties, as one can easily check by going through this whole section, even if this assumption does not make $\tilde{\partial}_{0}$ coincide with $\partial_{0}$.

\section{Dynamics of the Electromagnetic Field}

By introducing $\mathscr{E}^{T i} ; \mathscr{E}^{L i}=g^{\frac{1}{2}} g^{i j} e,{ }_{i} ; A_{i}^{T}$ and $A_{i}^{L} \equiv a,{ }_{i}$ into the action (8), using (13) and substituting $\mathscr{F}_{i j}=g^{\frac{1}{2}}\left(\partial_{i} A_{j}-\partial_{j} A_{i}\right)$, we obtain:

$$
\begin{aligned}
& I^{\text {e.m. }}\left(g_{i j}, n, N^{i}, \mathscr{E}^{T i}, \mathscr{E}^{L i}, A_{i}^{T}, A_{i}^{L}, \tilde{A}^{0}\right) \\
& =\int\left\{-\left(\mathscr{E}^{T i}+\mathscr{E}^{L i}\right) \tilde{\partial}_{0} A_{i}^{T}+\left(\tilde{A}^{0}+\tilde{\partial}_{0} a\right)\left[\partial_{i} \mathscr{E}^{L i}-\varrho\right]+j^{i} A_{i}^{T}-\frac{1}{2} g_{i j} g^{-\frac{1}{2}}\right. \\
& \left.\quad \cdot\left[\left(\mathscr{E}^{T i}+\mathscr{E}^{L i}\right)\left(\mathscr{E}^{T j}+\mathscr{E}^{L j}\right)+\mathscr{B}^{i} \mathscr{B}^{j}\left(A^{T}\right)\right]\right\} d \tilde{x}^{0} \wedge d^{3} \boldsymbol{x}
\end{aligned}
$$

(where $\mathscr{B}^{i}=\frac{1}{2} \varepsilon^{i j k} f_{j h}(g)^{-\frac{1}{2}}$ ).

In terms of these variables, gauge electromagnetic invariance of (17) means invariance of (17) under the transformation $(\chi$ is an arbitrary function)

$$
\begin{aligned}
& \mathscr{E}^{L i} \rightarrow \mathscr{E}^{\prime L i}=\mathscr{E}^{L i}, \quad \mathscr{E}^{T i} \rightarrow \mathscr{E}^{\prime T i}=\mathscr{E}^{T i}, \\
& A^{T}{ }_{i} \rightarrow A^{\prime T}{ }_{i}=A_{i}^{T}, \quad a \rightarrow a^{\prime}=a+\chi, \\
& \tilde{A}^{0} \rightarrow \tilde{A}^{0 \prime}=\tilde{A}^{0}-\tilde{\partial}_{0} \chi
\end{aligned}
$$

and is then trivial to check. The choice of a gauge means determining in a specific way the value of the longitudinal component of $A_{i}$. Consequently, as the gauge scalar $\alpha \equiv\left(\tilde{A}^{0}+\tilde{\partial}_{0} a\right)$ is determined by the field equations, once one chooses $\alpha, \tilde{A}^{0}$ is fixed. In fact, performing independent variations of $\alpha \mathscr{E}^{L i}=g^{i j} \partial_{j} e$ in the action (17) we obtain the equation for $\left(\tilde{A}^{0}+\tilde{\partial}_{0} a\right) \equiv \alpha$ :

$\delta I / \delta e=0 \rightleftarrows 2 n^{-1} \partial_{i}\left[n \hat{\pi}^{i j} A_{j}^{T}\right]+n^{-1} \partial_{i}\left[n \mathscr{E}^{i}\right]+n^{-1} \partial_{i}\left[g^{\frac{1}{2}} g^{i j} \partial_{j}(n \alpha)\right]=0$. 
Variations of either $a$ or $\tilde{A}^{0}$ only contribute to vary the Lagrange multiplier $\alpha$, so both give the same equation, which is the differential constraint of the system

$$
\partial_{i} \mathscr{E}^{i}=\partial_{i} \mathscr{E}^{L i} \equiv \partial_{i} g^{i j} g^{\frac{1}{2}} \partial_{j} e=\varrho .
$$

The dynamics is contained in the two sets of equations obtained after independent variations of $\left(A_{i}, \mathscr{E}^{j}\right)$ in (8) (which is equivalent to varying $A_{i}^{T}, \mathscr{E}^{T i}$ if (19) and (20) are taken into account),

$$
\begin{aligned}
& \delta I / \delta \mathscr{E}^{i}=0 \rightleftarrows \tilde{\partial}_{0} A_{i}=-g^{-\frac{1}{2}} \mathscr{E}_{i}-n^{-1} \partial_{i}\left[n \tilde{A}^{0}\right], \\
& \delta I / \delta A_{i}=0 \rightleftarrows \tilde{\partial}_{0} \mathscr{E}^{i}=-j^{i}-n^{-1} \partial_{j}\left[n \cdot f^{j i}\right] .
\end{aligned}
$$

Splitting $A_{i}$ as $\left(A_{i}^{T}+\partial_{i} a\right)$ and using $\left(14 \mathrm{a}^{\prime}\right)$, we find the evolution of the transverse electromagnetic potential:

$$
\tilde{\partial}_{0} A_{i}^{T}=-g^{-\frac{1}{2}} \mathscr{E}_{i}-n^{-1} \cdot \partial_{i}[n \alpha] .
$$

Introducing $\mathscr{E}_{i}$ into Eq. (21b), taking the value it has in Eq. (21a'), we arrive at the second-order propagation equation for the transverse electromagnetic potential $A_{i}^{T}$

$$
n^{-1} \partial_{j}\left[n f^{j i}\right]-\tilde{\partial}_{0}\left[g^{\frac{1}{2}} g^{i j} \tilde{\partial}_{0} A_{j}^{T}\right]=-j^{i}+\tilde{\partial}_{0}\left[n^{-1} g^{\frac{1}{2}} g^{i j} \partial_{j}(n \alpha)\right] .
$$

The first term on the left-hand side can be transformed by introducing the value of $f^{j i}=g^{\frac{1}{2}} g^{j h} g^{i k}\left[\partial_{h} A_{k}^{T}-\partial_{k} A_{h}^{T}\right]$ and using the law of interchange of covariant derivatives, i.e.,

$$
\left[D_{j}, D_{i}\right] A^{T j}=R_{i j} A^{T j} .
$$

In doing this, the first term becomes

$$
n^{-1} \partial_{j}\left[n f^{j i}\right]=g^{\frac{1}{2}}\left(\partial_{j} \log n\right)\left[D^{j} A^{T i}-D^{i} A^{T j}\right]+g^{\frac{1}{2}} \tilde{\Delta}_{j}^{i} A^{T j},
$$

where $\tilde{\Delta}_{j}^{i}$ is the De-Rham operator acting on a 3-vector which has been considered extensively by Lichnerowicz [5].

$$
\tilde{\Delta}_{j}^{i} A^{T j} \equiv D_{j} D^{j} A^{T i}-R_{j}^{i} A^{T j} .
$$

Substitution of the first term in Eq. (22) by its value (23b) gives for the propagation of $A_{i}^{T}$

$$
\begin{aligned}
g^{\frac{1}{2}} \hat{\square}^{i j}(g) A_{j}^{T} & \equiv g^{\frac{1}{2}} \tilde{\Delta}_{j}^{i} A^{T j}-\tilde{\partial}_{0}\left[g^{\frac{1}{2}} g^{i j} \cdot \tilde{\partial}_{0} A_{j}^{T}\right] \\
& =-j^{i}+g^{\frac{1}{2}}\left(\partial_{j} \log n\right)\left[D^{i} A^{T j}-D^{j} A^{T i}\right]+\tilde{\partial}_{0}\left[n^{-1} g^{\frac{1}{2}} g^{i j} \partial_{j}(n \alpha)\right],
\end{aligned}
$$

where the Lagrange electromagnetic multiplier $\alpha \equiv\left(\tilde{A}^{0}+\tilde{\partial}_{0} a\right)$ is determined by the constraint Eq. (19), which can be put in the more ex- 
plicit form:

$$
\begin{aligned}
2 n^{-1} \partial_{i}\left[n \hat{\pi}^{i j} A_{j}^{T}\right] & +\varrho-g^{\frac{1}{2}} g^{i j}\left(\partial_{i} \log n\right) \tilde{\partial}_{0} A_{j}^{T}-g^{\frac{1}{2}} g^{i j}\left(\partial_{i} \log n\right) n^{-1} \partial_{j}[n \alpha] \\
& +n^{-1} \partial_{i}\left[g^{\frac{1}{2}} g^{i j} \partial_{j}(n \alpha)\right]=0 .
\end{aligned}
$$

It should be noted that $j^{i}$ has to be understood in Eq. (24) as $j^{T i}+j^{L i}\left(j^{T}, \varrho\right)$ where $j^{L i} \equiv g^{\frac{1}{2}} g^{i j} \partial_{j} \beta$ is determined by Eq. (12), i.e.,

$$
n^{-1} \partial_{j} n g^{\frac{1}{2}} g^{i j} \partial_{j} \beta+\left(\partial_{j} \log n\right) j^{T j}+\tilde{\partial}_{0} \varrho=0 .
$$

Conversely to what happens in the flat case where the transverse modes decouple from the scalar mode $\left(-j_{i}^{L}+\partial_{0} \partial_{i} \alpha \equiv 0\right)$, the existence of a gravitational field avoids the disentangling of both modes and this is reflected in the non-vanishing of $-j^{L i}+\tilde{\partial}_{0} g^{\frac{1}{2}} g^{i j} n^{-1} \partial_{j} n \alpha$.

Actually, these problems already exist at the level of the first-order formalism and can be traced in the spreading over the space of longitudinal vectors of the variations in time of a pure transverse covariant vector $A_{i}^{T}$. The system (21), together with the constraint (19), can still be read:

$$
\begin{gathered}
\tilde{\partial}_{0} A_{i}^{T}=-g^{-\frac{1}{2}} \mathscr{E}_{i}^{T}-\partial_{i} e-n^{-1} \partial_{i} n \alpha, \\
\tilde{\partial}_{0} \mathscr{E}^{T i}=-j^{T i}-j^{L i}-\tilde{\partial}_{0} g^{\frac{1}{2}} g^{i j} \partial_{j} e-n^{-1} \partial_{j} n f^{j i}, \\
2 n^{-1} \partial_{i} n \hat{\pi}^{i j} A_{j}^{T}+\left(\partial_{i} \log n\right) \mathscr{E}^{T i}+n^{-1} \partial_{i} n g^{\frac{1}{2}} g^{i j} \partial_{j} e \\
\quad+n^{-1} \partial_{i} n g^{\frac{1}{2}} g^{i j} n^{-1} \partial_{j} n \alpha=0 .
\end{gathered}
$$

We assert that the contribution of the 2 longitudinal vectors in (25a) $\partial_{i}[e+\alpha]$ as well as the contribution of the 2 longitudinal pieces in $(25 b)$ $-\left(j^{L i}+g^{\frac{1}{2}} g^{i j} \partial_{j} \tilde{\partial}_{0} e\right)$ do not vanish just because of the coupling to the gravitational field and are not merely due to the kinematic distorsion caused by $n \neq$ const.

To see this, let us assume $n=1$. In this case $[e+\alpha]$ and $\left[\tilde{\partial}_{0} e+\beta\right]$ (which are determined by the constraints of the system) turn out to be, respectively, the non-null quantities

$$
\begin{aligned}
e+\alpha & =-2 \Delta_{g}^{-1} g^{-\frac{1}{2}} \partial_{i} \hat{\pi}^{i j} A_{j}^{T}, \\
\beta+\tilde{\partial}_{0} e & =2 \Delta_{g}^{-1} g^{-\frac{1}{2}} \partial_{i} \hat{\pi}^{i j} \partial_{j} e .
\end{aligned}
$$

\section{Gauge Independent Field Equations for the Transverse Modes}

In order to achieve the explicit time evolution of the transverse mode of the electromagnetic field, we shall often use the existence of the inverse $\Delta^{-1}(g)$ of the curved 3-laplacian elliptic operator acting on a 
scalar $f,[6]$

$$
\begin{aligned}
& \Delta(g) f \equiv g^{-\frac{1}{2}} \partial_{i} g^{\frac{1}{2}} g^{i j} \partial_{j} f, \\
& \Delta^{-1}(g) \Delta(g) f=f .
\end{aligned}
$$

Then the value of the coulombian mode of the electric vector, in view of Eq. (20), is given by

$$
e=\Delta^{-1}(g) g^{-\frac{1}{2}} \varrho,
$$

while from $\left(12^{\prime}\right)$ we obtain, for the potential of the longitudinal part of the current density, $j^{L i}$. To express $\beta$ as a function of the transverse current $j^{T_{t}}$ and the charge density, we have to make use of the technique of the conformal mappings of space-like three-geometries [6] to get rid of the dependence upon $n$. It is found that $\left(n^{2} g\right.$ means the tensor $\left.\left(n^{2} g\right)_{i j} \equiv n^{2} g_{i j}\right)$ :

$$
n^{2} g^{\frac{1}{2}} \Delta\left(n^{2} g\right) f \equiv n^{-1} \partial_{i} g^{\frac{1}{2}} n g^{i j} \partial_{j} f,
$$

and consequently $\beta$ is:

$$
\beta=-\Delta^{-1}\left(n^{2} g\right)\left[n^{-2} g^{-\frac{1}{2}}\left(\tilde{\partial}_{0} \varrho+j^{T i} \partial_{i} \log n\right)\right] .
$$

By dealing with Eq. $(25 \mathrm{c})$ in a similar way, we obtain the value of the scalar mode

$$
\alpha=-n^{-1} \Delta_{g}^{-1}\left\{n^{3} \Delta_{n^{2} g} \Delta_{g}^{-1}\left[g^{\frac{1}{2}} \varrho\right]+g^{-\frac{1}{2}} \mathscr{E}^{T i} \partial_{i} n+2 g^{-\frac{1}{2}} \partial_{i} n \hat{\pi}^{i j} A_{j}^{T}\right\} .
$$

Another useful relation is the explicit value of $\tilde{\partial}_{0} e$, which can be found by taking the time derivative of Eq. (20) and introducing, where necessary, the value of $e(28 \mathrm{a})$ :

$$
n \tilde{\partial}_{0} e=\Delta_{g}^{-1}\left\{g^{-\frac{1}{2}}\left[n \tilde{\partial}_{0} \varrho+2 \partial_{i} n \hat{\pi}^{i j} \partial_{j} \Delta_{g}^{-1} g^{-\frac{1}{2}} \varrho\right]\right\} .
$$

We note that (28d) does not depend upon either $A_{i}^{T}, \mathscr{E}_{i}^{T}$ or $\alpha$. The same property holds for $\beta$, as is shown by Eq. (28b), while, conversely, the scalar mode $\alpha$ is a functional of the 4 electromagnetic dynamical variables $\left(A_{i}^{T}, \mathscr{E}_{i}^{T}\right)$.

At this point we can reformulate the dynamics of the transverse modes (25) by introducing the values of $e, \beta$, a and $\tilde{\partial}_{0} e$ defined through Eqs. (28a)-(28d), respectively. Thus, the final equations for the physical electromagnetic fields a) in the presence of a source, b) in a complete 3-covariant fashion and c) gauge independent, are

$$
\begin{aligned}
\tilde{\partial}_{0} A_{i}^{T}= & -g^{-\frac{1}{2}} \mathscr{E}_{i}^{T}-\partial_{i} e(g, \varrho)-n^{-1} \partial_{i}\left[n \alpha\left(n, g, \pi, \varrho, \mathscr{E}^{T}, A^{T}\right)\right], \\
\tilde{\partial}_{0} \mathscr{E}^{T i}= & -j^{T i}-g^{\frac{1}{2}} g^{i j} \partial_{j} \beta\left(n, g, \varrho, j^{T}\right)-g^{\frac{1}{2}} g^{i j} n^{-1} \partial_{j}\left[n \tilde{\partial}_{0} e(n, g, \pi, \varrho)\right] \\
& +2 \hat{\pi}^{i j} \partial_{j}\left[\Delta_{g}^{-1} g^{-\frac{1}{2}} \varrho\right]-n^{-1} \partial_{j}\left[n g^{\frac{1}{2}} g^{j m} g^{i n}\left[\partial_{m} A_{n}^{T}-\partial_{n} A_{m}^{T}\right]\right] .
\end{aligned}
$$


Gravitationally coupled electromagnetic waves can be seen as excitation of the canonical variables $\left(A_{i}^{T}, \mathscr{E}_{i}^{T}\right)$. Because of the linearity of Eqs. (29) with respect to these variables, it is equivalent to considering the sourceless equations which, due to this, look simpler than the above set. In such a case they are:

$$
\begin{aligned}
& \tilde{\partial}_{0} A_{i}^{T}=-g^{-\frac{1}{2}} \mathscr{E}_{i}^{T}+n^{-1} \partial_{i} \cdot \Delta_{g}^{-1} \cdot g^{-\frac{1}{2}} \cdot\left[\mathscr{E}^{T i} \partial_{i} n+2 \partial_{i} n \hat{\pi}^{i j} A_{j}^{T}\right], \\
& \tilde{\partial}_{0} \mathscr{E}^{T i}=-n^{-1} \partial_{j} n g^{\frac{1}{2}} g^{j l} g^{i k}\left[\partial_{l} A_{k}^{T}-\partial_{k} A_{l}^{T}\right] .
\end{aligned}
$$

It is interesting to point out the choice of a specific gauge does not contribute to the structure of either set (29) or the set (30).

\section{Covariant Formulation of the Full Interacting System}

We can also formulate the gravitational action in a full covariant way by means of the covariant time derivative operator $\tilde{\partial}_{0}$. Starting from the reduced $6+6$ form of the action given by Arnowitt, Deser and Misner [2], discarding a total time derivative and a 3-divergence and introducing $\tilde{\partial}_{0}$, one obtains for the gravitational field:

$$
I^{G}\left(g_{i j}, \pi^{i j}, n, N^{i}\right)=\int\left\{\pi^{i j} \tilde{\partial}_{0} g_{i j}+g^{\frac{1}{2}}\left[{ }^{3} R\left(g_{i j}\right)+g^{-1}\left(\frac{1}{2} \pi^{2}-\pi^{i j} \pi_{i j}\right)\right]\right\} d \tilde{x}^{0} \wedge d^{3} \boldsymbol{x}
$$

(where the action depends upon $n$ through the square bracket times $d \tilde{x}^{0}$ and upon $N^{i}$ through $\tilde{\partial}_{0} g_{i j} d \tilde{x}^{0}$ ).

The total action for the gravitationally coupled electromagnetic field is the sum of (31) and the reduced electromagnetic action (17). Varying the gravitational variables $\left(\pi^{i j}, g_{i j}\right)$ in the total action $I \equiv I^{G}+I^{e m}$, we regain the Einstein equations for the gravitational field in a canonical and fully covariant fashion ${ }^{5}$

$$
\begin{aligned}
\delta I / \delta \pi^{i j}= & 0 \rightleftarrows g^{\frac{1}{2}} \tilde{\partial}_{0} g_{i j}=2 \pi_{i j}-\pi g_{i j}, \\
\delta I / \delta g_{i j}= & \rightleftarrows \rightleftarrows \tilde{\partial}_{0} \pi^{i j}=-g^{\frac{1}{2}} G^{i j}(g)+g^{-\frac{1}{2}}\left[\pi \pi^{i j}-2 \pi^{i} \pi^{\cdot j}-\frac{1}{2} g^{i j}\left(\frac{1}{2} \pi^{2}-\pi^{\cdot x} \pi \cdot x\right)\right] \\
& +g^{\frac{1}{2}}\left[g^{i l} g^{j h}-g^{i j} g^{l h}\right] n^{-1} D_{l} D_{h} n \\
& -\frac{1}{2} g^{-\frac{1}{2}}\left[\left(\mathscr{E}^{T i}+\mathscr{E}^{L i}\right)\left(\mathscr{E}^{T j}+\mathscr{E}^{L j}\right)+\mathscr{B}^{i} \mathscr{B}^{j}-\frac{1}{2} g^{i j}\left(\mathscr{E}^{l} \mathscr{E}_{l}+\mathscr{B} \mathscr{B} .\right)\right] .
\end{aligned}
$$

The gravitational constraints arise from independent variations of $\left(n, N^{i}\right)$. Variations of $n$ yield the 0 -constraint:

$$
g^{\frac{1}{2}}{ }^{3} R+g^{-\frac{1}{2}}\left(\frac{1}{2} \pi^{2}-\pi^{\cdot x} \pi \cdot x\right)+j^{i} A_{i}^{T}=\frac{1}{2} g^{-\frac{1}{2}}(\mathscr{E} \cdot \mathscr{E} .+\mathscr{B} \mathscr{B} .),
$$

while variations of $N^{i}$ give the vectorial constraint:

$$
2 D_{l} \pi_{j}^{l}=\varrho A_{j}^{T}-\mathscr{E}^{i} \partial_{[j} A_{i]}^{T}=\varrho A_{j}^{T}-\varepsilon_{j l m} \mathscr{E}^{l} \mathscr{B}^{m} \equiv \mathscr{S}_{j} .
$$

5 Although variations of $g_{1 j}$ include in principle variations of $-\mathscr{E}^{L i}=g^{\frac{1}{2}} g^{l_{j}} \hat{\partial}_{j} e$ and $A^{T} i$ as functionals of $g_{i j}$ those do not contribute by virtue of the Maxwell Field equations. 
Of course, the remaining equations are (20) and (25). Notice that $\mathscr{S}_{j}$ does not contain $g_{i j}$. Eq. (33) are not concerned with the time derivatives and so they have to be the same that appear in A.D.M. paper although (30) looks like very different.

In view of the simple form taken by the constraints (33) and the $\dot{p}=f(p, q)$ Eq. (32b), Deser gave [3] an alternative formulation for the system assuming, as principal variables, the two symmetric tensors $\left(\pi^{i j}, R_{i j}\right)$ instead of the natural choice $\left(\pi^{i j}, g_{i j}\right)$. As we are working in a full-covariant way using the "covariant" time derivative $\tilde{\partial}_{0}$, we need only evaluate $\tilde{\partial}_{0} R_{i j}$ to obtain the new set of equations. This can be done by means of the Palatini identity for the Ricci tensor. This identity yields the change $\delta R_{i j}$ of the Ricci tensor when the metric $g_{i j}$ changes $\delta g_{i j}$. In the present situation we take $\delta g_{i j}=\tilde{\partial}_{0} g_{i j} d \tilde{x}^{0}$, and consequently $\delta R_{i j}$ is $\delta R_{i j}=\tilde{\partial}_{0} R_{i j} d \tilde{x}^{0}$, without any sort of restriction on the co-ordinates we are dealing with.

For arbitrary variations $\delta g_{i j}$, the Palatini identity reads:

$$
2 \delta R_{i j}=-\Delta \delta g_{i j}+D_{k i} \delta g_{j}^{k}+D_{k j} \delta g_{i}^{k}-D_{i j} \delta g_{k}^{k} .
$$

Substituting $\delta R_{i j}$ by $\tilde{\partial}_{0} R_{i j} d \tilde{x}^{0} ; \delta g_{i j}$ by $\tilde{\partial}_{0} g_{i j} d \tilde{x}^{0}$, and introducing (32a) to eliminate these last time derivatives we obtain for the time evolution of $R_{i j}$,

$$
g^{\frac{1}{2}} \tilde{\partial}_{0} R_{i j}=-\Delta \pi_{i j}+\frac{1}{2}\left(g_{i j} \Delta-D_{i j}\right) \pi+D_{k i} \pi_{j}^{k}+D_{k j} \pi_{i}^{k} .
$$

As the constraints (33b) give the value of the divergence of $\pi_{i j}$ and as we cannot introduce this value directly into Eq. (34b) because covariant differential operators acting on a symmetric tensor do not commute, it is convenient to give another expression by interchanging the differential operators in the last term. Taking into account the specific relation which exists between the Riemann tensor and the Ricci tensor in a three-dimensional space, i.e.,

$$
R_{i j k l}=g_{i l} R_{j k}-g_{j l} R_{i k}+g_{j k} R_{i l}-g_{i k} R_{j l}-\frac{1}{2} R\left(g_{i l} g_{j k}-g_{j l} g_{i k}\right),
$$

and the two relations which arise from contracting Eqs. (32a) and (34b) with the metric tensor

$$
\begin{aligned}
g_{i j} \tilde{\partial}_{0} \pi^{i j}= & \frac{1}{2} g^{-\frac{1}{2}}(\mathscr{E} \mathscr{E} .+\mathscr{B} \mathscr{B} .)-\frac{1}{2} j^{i} A_{i}^{T}, \\
g^{\frac{1}{2}} g^{i j} \tilde{\partial}_{0} R_{i j}= & 2 D^{i} D_{j} \pi_{i}^{j}=2 D^{i} \mathscr{S}_{i}=D^{i} \cdot \varrho A_{i}^{T}-\mathscr{E}^{i} \tilde{\Delta}_{i j} A^{T j} \\
& +D^{i} \mathscr{E}^{j}\left[D_{j} A_{i}^{T}-D_{i} A_{j}^{T}\right],
\end{aligned}
$$

one obtains for the evolution of the three-Einstein tensor:

$$
\begin{aligned}
g^{\frac{1}{2}} \tilde{\partial}_{0} G^{i j}= & -\Delta_{g} \pi^{i j}+\frac{1}{2}\left(g^{i j} \Delta_{g}-D^{i j}\right) \pi+D^{i} \mathscr{S}^{j}+D^{j} \mathscr{S}^{i}-g^{i j} D_{l} \mathscr{S}^{l} \\
& +\left(\pi^{i l} G_{l}^{j}+\pi^{j l} G_{l}^{i}\right)-2 G \pi^{i j}+g^{i j}\left(\pi G-\pi^{\cdot x} G_{\cdot x}\right) .
\end{aligned}
$$


In a recent letter York [6] introduced the conformal part of the spatial metric tensor $R_{i j k}$ to study the gravitational degrees of freedom and the initial value problem in the case of a sourceless gravitational field. Finding attractive the idea of splitting the spatial metric into its conformal part $\bar{g}_{i j}$ and a conformal factor $\varphi^{4}$ which determines it $g_{i j}=\varphi^{4-} g_{i j}$, we shall evaluate the evolution of the conformal part. (As we shall see, the factor $\varphi^{4}$ is fixed by the 0 -contraint (33a).)

The 3-dimensional conformal tensor $R_{i j k}$ is defined by:

$$
R_{i j k} \equiv D_{k} R_{i j}-D_{j} R_{i k}+\frac{1}{4}\left(g_{i k} D_{j}-g_{i j} D_{k}\right) R \text {. }
$$

Variations of the metric tensor $g_{i j}=\tilde{\partial}_{0} g_{i j} d \tilde{x}^{0}$ entail corresponding variations of the conformal tensor $\delta R_{i j k}=\tilde{\partial}_{0} R_{i j k} d \tilde{x}^{0}$ which can be evaluated by means of Eqs. (32a), (34b), (35) and the knowledge of $\delta \Gamma_{i j}^{l}=\frac{1}{2}\left(D_{i} \delta g_{j}^{l}+D_{j} \delta g_{i}^{l}-D^{l} \delta g_{i j}\right)$. Thus, one obtains for $\delta R_{i j k}$,

$$
\begin{aligned}
\delta R_{i j k}= & D_{k} \delta R_{i j}-D_{j} \delta R_{i k}+\frac{1}{4}\left(g_{i k} D_{j}-g_{i j} D_{k}\right) \delta R \\
& +\frac{1}{4}\left(\delta g_{i k} D_{j}-\delta g_{i j} D_{k}\right) R+\delta \Gamma_{i j}^{l} R_{l k}-\delta \Gamma_{i k}^{l} R_{l j},
\end{aligned}
$$

which shows that the time evolution is given by

$$
\begin{aligned}
\tilde{\partial}_{0} R_{i j k}= & D_{k} \tilde{\partial}_{0} R_{i j}-D_{j} \tilde{\partial}_{0} R_{i k}+\left(g_{i k} D_{j}-g_{i j} D_{k}\right) \tilde{\partial}_{0} R+\frac{1}{4}\left(\tilde{\partial}_{0} g_{i k} D_{j}-\tilde{\partial}_{0} g_{i j} D_{k}\right) R \\
& +\frac{1}{2} R_{k}^{l}\left(D_{i} \tilde{\partial}_{0} g_{j l}+D_{j} \tilde{\partial}_{0} g_{i l}-D_{l} \tilde{\partial}_{0} g_{i j}\right) \\
& -\frac{1}{2} R_{j}^{l}\left(D_{i} \tilde{\partial}_{0} g_{k l}+D_{k} \tilde{\partial}_{0} g_{i l}-D_{l} \tilde{\partial}_{0} g_{i k}\right) .
\end{aligned}
$$

Inserting $\tilde{\partial}_{0} R_{i j}=-\Delta_{g} \pi_{i j}+\frac{1}{2}\left(g_{i j} \Delta_{g}-D_{i j}\right) \pi+3\left(\pi_{i l} R_{j}^{l}+\pi_{j l} R_{i}^{l}\right)$ $+R\left(g_{i j} \pi-\pi_{i j}\right)-2 g_{i j} \pi_{\cdot x} R^{\cdot x}-2 \pi R_{i j}+D_{i} \mathscr{S}_{j}+D_{j} \mathscr{Y}_{i}$ and $\tilde{\partial}_{0} g_{i j}$ into this formula we get for the evolution of the 3-conformal tensor

$$
\begin{aligned}
g^{\frac{1}{2}} \tilde{\partial}_{0} & R_{i j k}=D_{j} \Delta_{g} \pi_{k i}-D_{k} \Delta_{g} \pi_{j i}+\frac{1}{2}\left[D_{j}, D_{k}\right] D_{i} \pi+\frac{1}{2}\left[g_{i j} D_{k}-g_{i k} D_{j}\right] \Delta_{g} \pi \\
& +R_{k l}\left[D_{i} \pi_{j l}-2 D_{j} \pi_{i l}-D_{l} \pi_{i j}\right]-R_{j}^{l}\left[D_{i} \pi_{k l}-2 D_{k} \pi_{i l}-D_{l} \pi_{i k}\right] \\
& +3 R_{i}^{l}\left[D_{k} \pi_{j l}-D_{j} \pi_{k l}\right]-R\left[D_{k} \pi_{i j}-D_{j} \pi_{i k}\right]+\frac{1}{2}\left[g_{i j} R_{k}^{l}-g_{i k} R_{j}^{l}\right] D_{l} \pi \\
& +3 \pi_{i l}\left[D_{k} G_{j}^{l}-D_{j} G_{k}^{l}\right]+3\left[\pi_{j l} D_{k}-\pi_{k l} D_{j}\right] R_{i}^{l}+\frac{3}{2}\left[g_{i k} D_{j}-g_{i j} D_{k}\right] \pi_{\cdot} R^{\cdot x} \\
& -\frac{3}{2}\left[G_{i j} D_{k}-G_{i k} D_{j}\right] \pi-2 \pi\left[D_{k} G_{i j}-D_{j} G_{i k}\right]+\frac{1}{2}\left[g_{i k} D_{j}-g_{i j} D_{k}\right] D^{l} \mathscr{S}_{l} \\
& +\left[D_{k i} \delta_{j}^{l}-D_{j i} \delta_{k}^{l}+D_{k j} \delta_{i}^{l}-D_{j k} \delta_{i}^{l}\right] \mathscr{S}_{l} .
\end{aligned}
$$

Also in this case the requirement that $\pi^{i j}$ be traceless [7] $(\pi=0)$ allows us to solve the problem of the initial conditions, assuming the conformal law of transformation: $\bar{g}_{i j} \rightarrow \varphi^{4} \bar{g}_{i j}=g_{i j}, \bar{\pi}^{i j} \rightarrow \varphi^{-4} \bar{\pi}^{i j}=\pi^{i j}$ given by York. To do this, one gives an arbitrary metric $\bar{g}_{i j}$ and constructs a symmetric density $\bar{\pi}^{i j}$ so that, besides being traceless $(\bar{\pi}=0)$, verifies the vectorial constraint (33b) with respect to the given metric, namely

$$
\bar{D}_{j} \bar{\pi}_{i}^{j}=\bar{D}_{j}\left[\bar{g}_{i l} \bar{\pi}^{j l}\right]=\mathscr{S}_{i} .
$$


Then we must determine $\varphi$ in order to have a complete set of initial conditions $\left\{g_{i j}=\varphi^{4} \bar{g}_{i j}, \pi^{i j}=\varphi^{-4} \bar{\pi}^{i j}\right\}$ which still verify the traceless character of $\pi^{i j}(\pi=0)$.

In fact, under the conformal transformation $C, \bar{\pi}_{j}^{i}$ is a scalar quantity,

$$
\bar{\pi}_{j}^{i} \rightarrow \pi_{j}^{i}=\bar{\pi}_{j}^{i},
$$

and, moreover, the transformed $\pi_{j}^{i}$ solves the vectorial constraint \{because $\pi=\bar{\pi}=0$ \}

$$
\bar{D}_{j} \bar{\pi}_{i}^{j}=D_{j} \pi_{i}^{j}=\mathscr{S}_{i}
$$

Then, if one introduces $g_{i j}=\varphi^{4} \bar{g}_{i j}$ into the scalar constraint (33a), one obtains an elliptic equation which fixes $\varphi$. In the present case it turns out to be:

$$
\begin{aligned}
\bar{\Delta} \varphi=\frac{1}{8} \bar{R} \varphi+\frac{1}{8} \varphi^{-1} \cdot \bar{g}^{-\frac{1}{2}} \cdot j^{i} A_{i}^{T} & -\frac{1}{16} \varphi^{-3} \bar{g}^{-1}\left[\mathscr{E}^{i} \mathscr{E}^{j}+\mathscr{B}^{i} \mathscr{B}^{j}\right] \bar{g}_{i j} \\
& -\frac{1}{8} \varphi^{-7} \bar{g}^{-1} \bar{\pi}_{j}^{i} \bar{\pi}_{i}^{j}(\mathscr{S}) .
\end{aligned}
$$

\section{Applications}

We shall apply the present formalism to prove that a sourceless $\left(\mathscr{I}^{\mu}=0\right)$, merostatic, asymptotically flat-null electromagnetic-gravitational interacting system consists of a Lorentz flat 4-space and a null maxwellian field. By merostatic system we mean an electromagnetic gravitational system such that there exists some family of hypersurfaces where it is verified that $\tilde{\partial}_{0} A_{i}^{T}=0=\tilde{\partial}_{0} g_{i j}$ in the whole 4-space.

It will be sufficient to see, that the electromagnetic field vanishes because, if this is so, the problem has been reduced to the pure gravitational and sourceless case, and then we can follow essentially the same argument given by Arnowitt and Deser [8], although ours will differ slightly.

The key to this result is a theorem given by Lichnerowicz [9] for elliptic operators on a riemannian space which says that a harmonic function $u\left(\Delta_{g}(u)=0\right)$ which converges uniformly to a constant $k$ in the infinity of an elliptic riemannian manifold, coincides identically with this constant $(u \equiv k)$.

In the assumed merostatic conditions there is no $\mathscr{E}^{L i}(e=0)$ because we can apply to Eq. $(20)(\varrho=0)$ the above-mentioned theorem. As it follows from Eq. (32a) that $\pi_{i j}=0$, the Maxwell system (25) becomes:

$$
\begin{gathered}
0=\mathscr{E}^{T i}+g^{\frac{1}{2}} g^{i j} n^{-1} \partial_{j} n \alpha, \\
\tilde{\partial}_{0} \mathscr{E}^{T i}=-n^{-1} \partial_{j} n f^{j i}\left(A^{T}\right), \\
\mathscr{E}^{T i} \partial_{i} \log n+n^{-1} \partial_{i} n g^{\frac{1}{2}} g^{i j} n^{-1} \partial_{j} n \alpha=0 .
\end{gathered}
$$


Introducing $\mathscr{E}^{T i}$, given by (41a), into (41c), we find for the scalar mode $\alpha$,

$$
\partial_{i} g^{\frac{1}{2}} g^{i J} n^{-1} \partial_{j} n \alpha=0
$$

which, by making the conformal transformation $g_{i j} \rightarrow \bar{g}_{i j} \equiv n^{-2} g_{i j}$ achieves the final equivalent form:

$$
\Delta \bar{g}[n \alpha]=0
$$

where $\bar{g}_{i j}$ keeps the elliptic structure of $g_{i j}$. Then, again applying Lichnerowicz's theorem, we obtain the vanishing of $n \alpha$ and, consequently, of $\alpha$. Now, Eq. (41a) says that $\varepsilon^{T i}=0$. We have still to consider $A_{i}^{T}$, which now obeys

$$
\partial_{j}\left[n g^{\frac{1}{2}} g^{j i} g^{l h}\left(\partial_{i} A_{h}^{T}-\partial_{h} A_{i}^{T}\right)\right]=0
$$

This equation can be recast in the form:

$$
\tilde{\Delta}_{j}^{l} A^{T j}+\left[\left(D^{h} \log n\right) \delta_{j}^{l}-g^{h l} D_{j} \log n\right] D_{h} A^{T j}=0,
$$

which, by extension from the scalar case, we shall assume (together with the corresponding asymptotic conditions) gives uniquely $A_{i}^{T}=0$. Then, as the electromagnetic field vanishes, we are in the condition of a pure merostatic gravitational field which, as can easily be seen, is a flat 4-space. In fact, $\pi_{i j}$ implies, (33a), that the 3 -space curvature $R\left(g_{i j}\right)=0$.

Contracting the field Eq. (32b) we obtain for the last variable $n$ :

$$
\Delta_{g} n=0,
$$

and here again Lichnerowicz's theorem allows us to assert that $n \equiv 1$. Finally, instead of finding the hidden Lagrange multipliers $N^{i}$ we can go to the full Riemann tensor and prove its vanishing. As we have $K_{i j}=\pi_{i j}$ $=R_{i j}=0=n-1$, we can see that all its different components have already vanished. In fact, the Gauss-Codazzi equations give the vanishing of ${ }^{4} R_{i j k l},{ }^{4} R_{i j k}{ }^{\tilde{o}}$ (because $K_{i j}=0={ }_{3} R_{i j}$ )

$$
\begin{aligned}
& { }_{4} R_{i j k l}={ }_{3} R_{i j k l}+g^{-1} \cdot\left(K_{i k} K_{j l}-K_{i l} K_{j k}\right)=0, \\
& { }_{4} R_{i j l}{ }^{0}=D_{j} K_{i l}-D_{i} K_{j l}=0
\end{aligned}
$$

and because the last set of components of the 4-Riemann tensor are

$$
\left.{ }_{4} R_{t . j .}^{\tilde{0}} \tilde{0} .\right]_{k_{l \jmath}=0={ }_{3} R i j}=n^{-1} D_{i j} n,
$$

the value $n=1$ implies their vanishing and consequently the flatness of the 4-spaces with which we are dealing. 
Throughout all the sections we have seen the very different role played by $n$ compared with the other 3 -multipliers $N^{i}$. There is still another interesting property in connection with this point and this concerns the intrinsic definition of a static space. If one calculates the Killing tensor of the normal to $x^{0}=$ const. $\left(n_{\mu}=n \delta_{\mu}^{0}\right)$, one finds that $\Theta_{\mu \nu} \equiv D_{\mu} n_{v}+D_{v} n_{\mu}$ has the $3+1$ decomposition:

$$
\begin{aligned}
& \Theta_{i j}=-2 K_{i j} ; \quad \Theta^{\tilde{0} \tilde{0}}=0, \\
& \Theta_{i}^{\tilde{0}}=\partial_{i} \log n .
\end{aligned}
$$

Thus, $\pi_{i j}=0=\log n$ is equivalent to saying that $n_{\mu}$ (which by definition is hypersurface orthogonal) is a Killing vector, and then this coincides with the static case. However, $\pi_{i j}=0=K_{i j}$ alone does not imply the vanishing of the Killing tensor of $n_{\mu}$ (or the Killing tensor of any collinear vector) and the assumption of merostaticity is weaker than the assumption of staticity.

\section{Discussion}

We have obtained a $C$-formulation of the gravitationally coupled electromagnetic field by means of a $C$-time derivation generalizing previous results. This has allowed us to characterize the dynamical properties of the different variables involved in a total gauge independent way. We have seen the reasons for the mixing of transverse and longitudinal components of the fields, which are essentially dynamical as Eqs. (26) have shown and are not of merely kinematical origin ("bad" choice of co-ordinates). Then we have given the explicit formulation of the Maxwell field equations in terms of the $2+2$ canonical variables by means of the well-known inverse of the elliptic operator $\Delta(g)$ and conformal transformations. We have applied the full $C$-formulation of the interacting system to study the initial value problem where we determined the 3-conformal factor on the assumption of maximal embedding $(\pi=0)$. Finally, we have studied the sourceless-merostatic interacting system and the relation between merostatic and static fields. All results are given without any reference to the spatial Lagrange multipliers $N^{i}$, while the role played by $n$ is emphasised.

Acknowledgments. The author wishes to thank Professors Abdus Salam and Paolo Budini as well as the International Atomic Energy Agency and UNESCO, for hospitality at the International Centre for Theoretical Physics, Trieste.

The author also wishes to thank Professor Stanley Deser for bringing to his attention the problem of "curved" propagation of the electromagnetic field, and for fruitful criticism. 


\section{References}

1. Deser, S.: Lettere Nuovo Cimento 4, 1130 (1970).

2. Arnowitt, R., Deser, S., Misner, C. W.: Phys. Rev. 120, 313 (1960).

3. Deser, S. : Ann. Inst. Henri Poincaré 7, 149 (1967).

4. Fischer, A.E., Marsden, J.: University of California (Berkeley) preprint (1971).

5. Lichnerowicz, A.: Publ. Math. Inst. Hautes Etudes Sci. (Paris) 1961.

6. Bochner, S., Yano, K.: Curvature and Betti Numbers. Ann. Math. Stud. 32, (1953).

7. Lichnerowicz, A.: J. Math. Pure Appl. 23, 37 (1944). For more references and a recent application to the free gravitational field see J. W. York, Jr.: Phys. Rev. Letters 26, 1656 (1971) and J. Choquet-Bruhat, Commun. math. Phys. 21, 211 (1971).

8. DeWitt, B.: Phys. Rev. 160, 113 (1967).

9. Arnowitt, R., Deser, S. : Ann. Phys.(N.Y.) 23, 318 (1963).

10. Lichnerowicz, A.: Les théories relativistes de la gravitation et l'electromagnetisme, p. 135. Paris: Masson et Cie 1955.

C. Aragone

Instituto de Física

Facultad de Ingeniería

Universidad de la República

Herrera y Reissig 565

Montevideo, Uruguay 